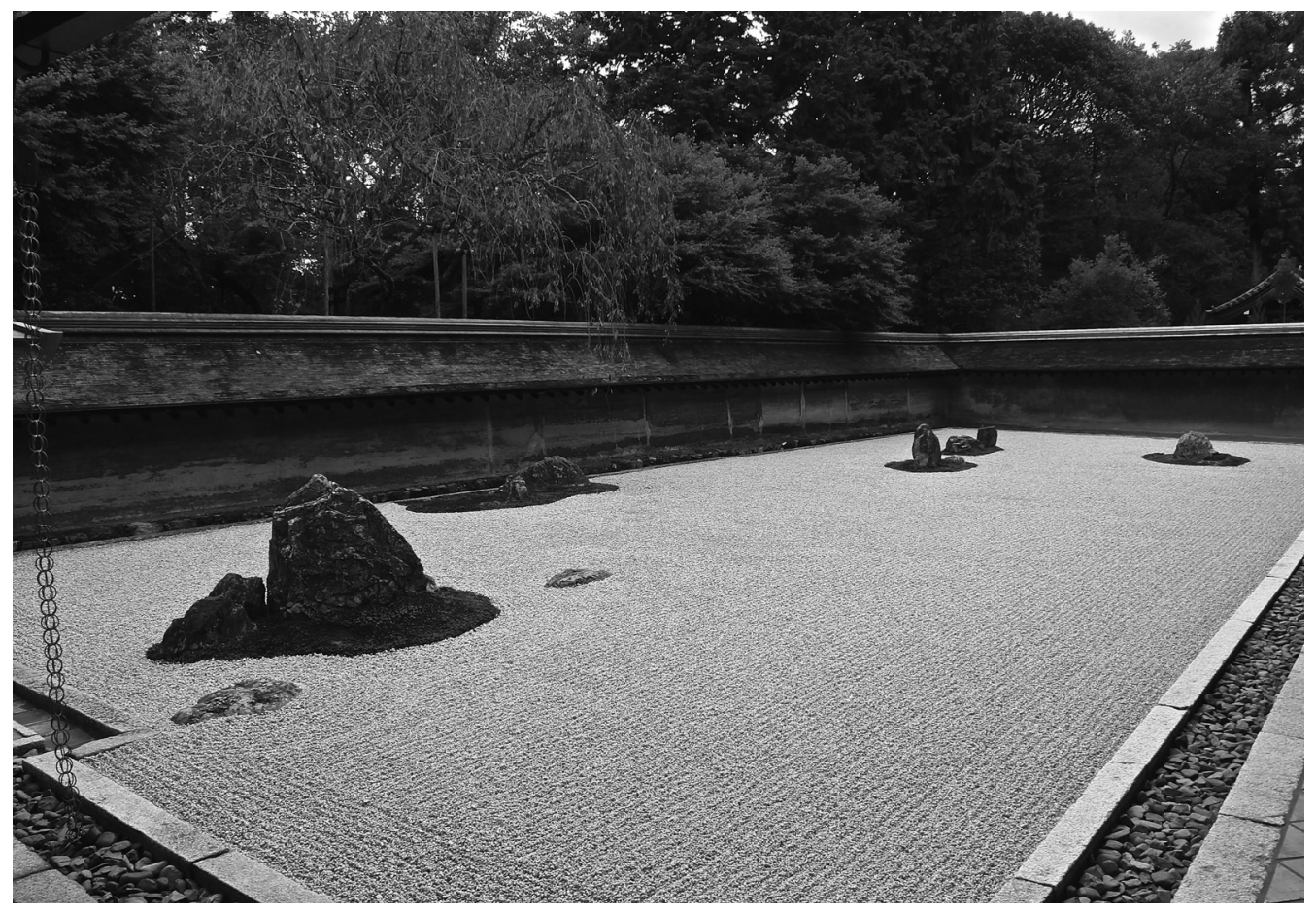

FIG. 1. Ryoan-ji (ca. 1400 d.C.)

\section{De los Silencios en el Jardín Seco Japonés}

\author{
Paz Soler Masota
}

Recibido 2016.08.08 :.: Aceptado 2016.08.16

DOI: 10.5821/palimpsesto. 15.4808

S ostenía Oscar WILDE que todo Japón es una pura invención ${ }^{1}$ Y tal vez estuviera en lo cierto, si se piensa que mientras en 1490 un bizarro Cristóbal Colón se refugiaba en La Rábida tras el rechazo inicial de los Reyes Católicos para financiar su aventura hacia las Indias, en Kyoto se gestaba el jardín seco o karesansui ${ }^{2}$ más enigmático de toda la Historia: Ryoan-ji (FIG. 1). Dos siglos antes ya se había escrito el Sakuteiki -uno de los dos grandes tratados de jardinería clásica japonesa- y levantado en Kennin-ji el primer templo zen donde se formó el gran maestro Muso Kokushi (también conocido como Soseki) bajo cuya luz se pergeñaron dos de los más icónicos jardines secos: el conocido como el Templo del Musgo (Kokedera o Saiho-ji) y el magnífico Tenryu-ji³.

Ryoan-ji sigue envuelto entre las brumas circundantes a su autoría. Se sostiene que fue obra de dos kawaramono, operarios de las castas inferiores y oficios más humildes que, con el tiempo, accedieron a mejor condición y dejaron su tímida firma en el envés de una de las rocas principales; trazos ya irreconocibles por desgastados. Sentencia del tiempo sobre la transitoriedad del hombre y de un paisaje que cada día es reconstruido devota y meticulosamente para ofrecerse ante la mirada de otros hombres que lo contemplarán de modo también fugaz.

El karesansui se sostiene sobre una cadencia de silencios que permiten una suerte de comunión plástica entre la obra y su espectador:

El primero de estos silencios es la aparente falta de rúbrica del artista jardinero, rasgo que contribuye al mejor ensalzamiento de la obra. La obra se pone a disposición del espectador sin imposición ni condicionante alguno, lo que impulsa un diálogo inmediato entre ambos. Este gesto es tanto gentil como consciente: habla del autor, sabedor de que su identidad deviene dato fútil y efímero, inscrita como está la obra en un entorno natura sometido a la implacable ley de la caducidad; de un autor que corrompe y manipula con destreza la naturaleza para evocar su esencia última -su carácter impermanente ${ }^{4}$ con la única intención de enfrentar al visitante a su mismo destino. Así pues, y aunque se conozca sobradamente su autoría (como ocurre en el mencionado Tenryu-ji, en la FIG. 2), la obra se presenta cual si fuera anónima, del mismo modo que será apreciada por quien nunca podrá ser su dueño: nadie podrá jamás adquirirla a título pleno, tan sólo poseerla mientras dure su experiencia al contemplarlo, que es a su vez una experiencia sensorial representativa del tránsito de lo mundano.

De la discreción propia del autor al respetuoso silencio del propio jardín frente a la Naturaleza que evoca. El jardín japonés se nutre del paisaje circundante con el que forma un continuo. Mediante la denominada técnica del shakkei (o paisaje tomado en préstamo), el karesansui se inscribe sutilmente en su entorno, al que se subordina para rendir tributo al discurso de las estaciones (F|G. 3).

\section{Sujeta como se halla a su temporalidad intrínseca, la} obra debe reverdecerse de modo constante; de lo contrario perecerá como tal, salvo en la mirada pretérita del viajero. Urgida de perseverante renovación, la obra se convierte en inacabada por naturaleza, debatiéndose paradójicamente entre la inmanencia como ideal inalcanzable y la imperfección como trasunto de lo real. A lo largo de su cíclica existencia, reproducción tras reproducción, el jardín seco japonés tal vez nos parezca inmutable, imperturbable o incluso estático. Mas si ésta fuera nuestra percepción última, como nos amonestaría Oscar WILDE, sencillamente no habríamos entendido nada. La falta de variación es instrumental: alude al carácter ilimitado de la repetición y, con ella, sirve al propósito de exaltar la identidad más profunda de lo evocado, de generar una disposición reverencial hacia lo natural. La perfección del lugar tanto como la imperfección de cada individuo. Cada día, la repetición transforma lo presente en algo ya pasado, manteniéndose la impresión de una presencia ausente y, así, el jardín se alza como un ámbito de resonancia que alberga algo que no admite explicación ni representación: su propia memoria. El karesansui solemniza un método de medición del tiempo y el fenecer de las cosas en el que lo único permanente es el cambio.

Tercer silencio apenas anunciado: el elogio de la imperfección a través de la renuncia a la geometría. Admiro el jardín japonés porque trasciende la geometría y se ocupa de la metafísica de la naturaleza, declaraba Isamu NOGUCHI ${ }^{5}$. Asimetría y vacío como reflejo del caos originario e indistinción de la creación primera del cosmos, como la expresión misma de lo eternamente cambiante
En rigor, la asimetría no es la finalidad directamente perseguida por el artista, sino el resultado de una composición ponderada que atiende al contrapeso adecuado entre el espacio lleno y el vacío (FIG. 4). Conceptualmente, pues, la asimetría es el arquetipo del equilibrio más afinado. La supuesta imperfección a nuestros ojos occidentales es pretendida en Japón de modo plenamente consciente.

Curiosamente, la asimetría se incorpora como tal parámetro estético en la tradición japonesa gracias al jardín: el estilo arquitectónico del shinden-zukuri a partir del cual se edificaron desde el período Heian las residencias y los templos nunca fue seguido en el espacio dedicado al jardín, sentándose desde el principio la regla de su composición asimétrica ${ }^{7}$. De hecho, el Sakuteiki contiene abundantes referencias a la colocación de los elementos del jardín exhortando a huir de la simetría o, si se prefiere, a acoger desde el principio una cierta heterodoxia que, en buena lógica, habría de permitir al artista expresarse con una mayor libertad ${ }^{8}$.

Sobre lo anterior, la asimetría contribuye a dotar de dinamismo a la composición así como de una mayor expresión de infinitud, como nos desvelaba con bellas palabras Yasunari KAWABATA:

El jardín japonés también simboliza la vastedad de la naturaleza. Mientras el jardín occidental tiende a ser simétrico, el jardín japonés es asimétrico, porque lo asimétrico tiene mayor fuerza para simbolizar lo múltiple y lo vasto. Esta asimetría, desde luego, se apoya en el equilibrio impuesto por la delicada sensibilidad del hombre japonés. De allí que nada sea tan complicado, variado, atento al detalle, como el arte de la jardinería japonesa'

Y la anti-representación como cuarto silencio. Por contraste con los anteriores paisajes de índole alegórica durante el periodo Heian, en el karesansui no se halla ni rastro de mímesis. Es la ausencia -alusiva a la presenciaun rasgo conceptual del jardín karesansui, el que supone el salto estético respecto de sus antecesores ${ }^{10}$.

Ello entronca, a su vez, con el gusto japonés por lo no evidente, por lo sugerido, lo traslúcido ${ }^{11}$

Para leer un karesansui, es necesario analizar la disposición abstracta de las piedras y captar la complejidad sincrética del conjunto, así como el flujo temporal que de todo ello emana ${ }^{2}$.

Mediante la denominada técnica del shakkei (o paisaje tomado en préstamo), el karesansui se inscribe sutilmente en su entorno, al que se subordina para rendir tributo al discurso de las estaciones.

Pero la abstracción no entendida como un abandono forzoso de la materialidad de lo representado, sino de una encarnación del valor intrínseco del motivo. Cada rasgo en la obra se subordina a la objetividad de la manifestación artística, que comunica la versión más fidedigna de las cosas, del modo más sencillo posible. $Y$, sin embargo, no se pretende trasladar una mera imagen del motivo, sino su esencia, su ser, su existencia metafísica. Tal vez por ello la comprensión de estas obras se haya mantenido perdurable en el tiempo.

En un karesansui, esta esencialidad informa también la renuncia a la materialidad expresa sin pérdida alguna de significado. En efecto, no sólo existe una alusión abstracta del motivo completo, sino también una simplificación manifiesta de la relación interna que cohesiona los elementos de la composición. Pero de nuevo, materialidad o abstracción no son polos opuestos que se excluyan mutuamente: no existe contradicción impropia del ser, que desde el principio concilia y sobrevuela por encima de esta presunta dualidad.

Asimetría, ligereza, elogio del tiempo, refinada profundidad y quietud. Mas todo ello no para el disfrute estético, sino como presupuesto para entrenar la mente del hombre y conducirle a la realidad última. Según el maestro Daisetz T. SUZUKI, ésta era la finalidad de 

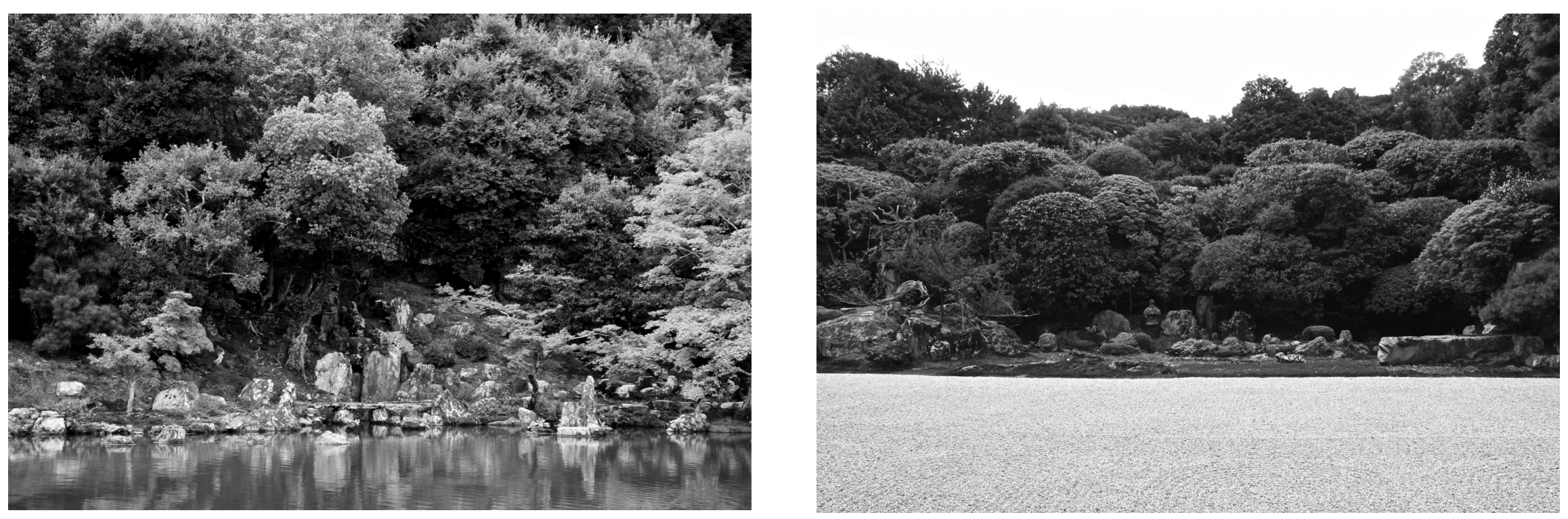

FIG. 2. Tenryu-ji (ca. 1339 d.C.

$\wedge$ FIG. 3. Konchi-An (ca. 1579 d.C.)

Cada día, la repetición transforma lo presente en algo ya pasado, manteniéndose la impresión de una presencia ausente y, así, el jardín se alza como un ámbito de resonancia que alberga algo que no admite explicación ni representación: su propia memoria.

las artes expresivas del zen. $Y$ aunque el karesansui constituya un género harto más complejo, es innegable la influencia del zen en su concepción sublime de lo austero, en su calma belleza de la negación.

Gracias a sus silencios, el espectador se inscribe física y anímicamente en la apreciación de la esencia del jardín, la que reside más allá de las formas, incluso del espacio y del tiempo. Cuando la emoción trasciende la admiración de su belleza extrínseca, el karesansui habrá cumplido su razón de ser: acoger al visitante como su huésped eventual, pasajero como es de su propia existencia: Ars longa, vita brevis. Y, sin embargo, la contemplación de la obra que sabemos impermanente nos procura un sorbo de eternidad.

1 En su ensayo La Decadencia de la Mentira (The Decay of Lying, 1891), cita extraida de MARCH, PH./DELANK, C. (eds.), The Adventure of Japanese Photography 1860-1890, Heidelberg (Kehrer Verlag) 2002, p. 7, el autor sostenía que nuestro espejismo del mundo es más fuerte que la realidad: " $E$ En serio imaginan Uds. verdaderamente que los japoneses son tal como se nos presentan en el arte, que existen de algún modo? Si lo hacen, es porque nunca han entendido el arte japonés. Los japoneses son la creación deliberadamente consciente de ciertos artistas. Si contemplamos una imagen de Hokusai o Hokkel, o de algun gran pintor nativo junto a una dama o un caballero reales, observarán que no existe entre ambos ni la más minima semblanza. La gente real que vive en Japón no es distinta del común de los ingleses: lo que es decir que son extremadamente lugar común, y no cabe predicar nada curioso o extraordinario sobre ellos. De hecho, todo Japón es una pura invención. No existe tal país, no existe tal pueblo".

${ }^{2}$ La etimología de la expresión karesansui ha sido objeto de profundo estudio. Conformada esencialmente por la comunión jardin, donglas, sansui consthyouna sinecdoque alusiva al jardin, donde san representa a la montaña y sui al agua. El jardín, puerm, conte con lo fluido y transtorio. De su parte ke soldoy las extensiones de grava 0 aren blancas propia de los templos sintoístas y, más tarde, zen.

${ }^{3}$ En general y entre nosotros VIVES, J., Historia y Arte del jardín japonés, Gijón (Satori) 2014.

${ }^{4}$ Véase la editorial firmada por Alberto PEÑín en el número 4 de Palimpsesto, 2014, Lo único que permanece es el cambio, p. 2, en el mismo el trabajo de FERRANDO, J., Arquitectura es Paisaje. Una expedición a la arquitectura como punto de encuentro.

${ }^{5}$ En CANOGAR, S., Cuando el cerezo florece, en GRAS, M. (Coord.), El Jardín Japonés. Qué es y no es entre la espacialidad y la temporalidad del paisaje, Madrid (Tecnos), 2015, pp. 369 y ss., p. 377 . Asimetria representada en la 'pérdida de centro' característica en la arquitectura japonesa: ÁLVAREZ, EI Karesansui moderno: Mirei Shigemori e Isamu Noguchi, ob. cit. ult., pp. 457 y ss., p. 459, siguiendo a Tadao ANDO: "(E)n contraposición a la cultura occidental, el centro del jardín intensifica esa congelación del tiempo. La distancia entre las piedras se convierte también en una medida de tiempo".

${ }^{6}$ Vid. GARCÉS, Joshia Conder y la Estética Demiurga del Jardín Japonés, ob. cit. ult., pp. 439 y ss., pp. 447,449 y 452.

${ }^{7} \mathrm{Y}$, desde luego, el primer karesansui conocido, la cascada seca atribuida a Muso SOSEKI en Saiho-ji, ya era notoriamente asimétrico, como el propio jardín del templo: vid. RODRíGUEZ Taut y la modernidad de la villa Katsura, en GRAS, EI Jardín Japonés, ob. cit., pp. 519 y ss., p. 534

${ }^{8}$ Conforme NAKAGAWARA, The Japanese Garden for the Mind: The 'Bliss' of Paradise Trascended, Stanford Journal of East Asian Affairs, vol. 4, num. 2, 2004, pp. 83 y ss., p. 89. Desde una perspectiva contemporanea, conforme con este principio se hala James C. ROSE, Freedom in the Garden, en TREIB, M. (ed), Modern Landscape Architecture: A Critical Review, Cambridge, Mass.-London (MIT Press) 1992, en p. 69: "Why should we design gardens or even world fairs like Le Nôtre? No one would think of furnishing a room on the principle of the axis. You do not expect to stand at one end and find an aesthetic composition at the other. You want a sense of proper division and interest from any point. So with gardens: it is fundamentally wrong to begin with axes or shapes in plan; ground forms evolve from a division

9 En su conferencia Japan, the Beautiful and Myself, al aceptar e Nóbel de Literatura el 10 de diciembre de 1968.

${ }^{10}$ Cfr. entre nosotros CARBONELL, O., Paraísos Occidentales, Espacios Universales: simbología y representación del jardín japonés, en GRAS, El Jardín Japonés, ob. cit., pp. 193 y ss. p. 199

${ }^{11}$ Cfr. PIZARRO, Un jardín japonés: topografías del vacío, ob. cit. ult., pp. 425 y ss., p. 432, siguiendo a RUIZ DE LA PUERTA.

${ }^{12}$ Cfr. Richard SERRA en CHILLIDA, en WAA, Variaciones sobre el jardín japonés, Madrid (La Casa Encendida) 2014, p. 31.
ABSTRACT

El término Karesansui alude a un género singular de jardines japoneses compuesto de rocas, gravilla y arena, con obras artísticas tan sublimes como el renombrado Ryoan-j. De gestos entrelazados sobre el silencio, el jardín genera una comunión plástica con quien lo contempla: emplazado ante un escenario donde la Naturaleza representa el devenir de las estaciones, el hombre es confrontado con la emoción de su propia existencia efímera.

Umbral de estos silencios es la tenue huella de la autoría del artista, consciente y humilde reconocimiento de la condición humana. Y así también el jardín, urgido de renovación constante para sobreponerse a su natural declive, se revela como inacabado e imperfecto, solemnizando con ello un método de medición del tiempo en el que lo único permanente es el cambio. De ahí que el jardín renuncie silente a la geometría, para hilar con asimetría y vacío el más refinado equilibrio en expresión de infinitud. Y la anti-representación configurando el cuarto silencio: la abstracción como encarnación del valor intrínseco del motivo, de su esencia misma, de su existencia metafísica.

Merced a sus silencios, el visitante ingresa en la apreciación de jardín que lo acoge como su huésped temporal en un espacio que, no obstante su carácter impermanente, nos procura, espectadores transeúntes como somos del mismo, un sorbo de eternidad.

PALABRAS CLAVE: Karesansui, Jardines Japoneses, Zen Ryoanji.

PAZ SOLER MASOTA es Doctora en Bellas Artes por la Universidad de Barcelona y profesora titular de Derecho Mercantil en la Universitat Pompeu Fabra.

El artículo revisa parte de la investigación desarrollada por la autora para su tesis doctoral, defendida en febrero de 2016 ante un tribunal compuesto por los Doctores Carlos Ferrater, Alberto Peñín y Gloria Moure.

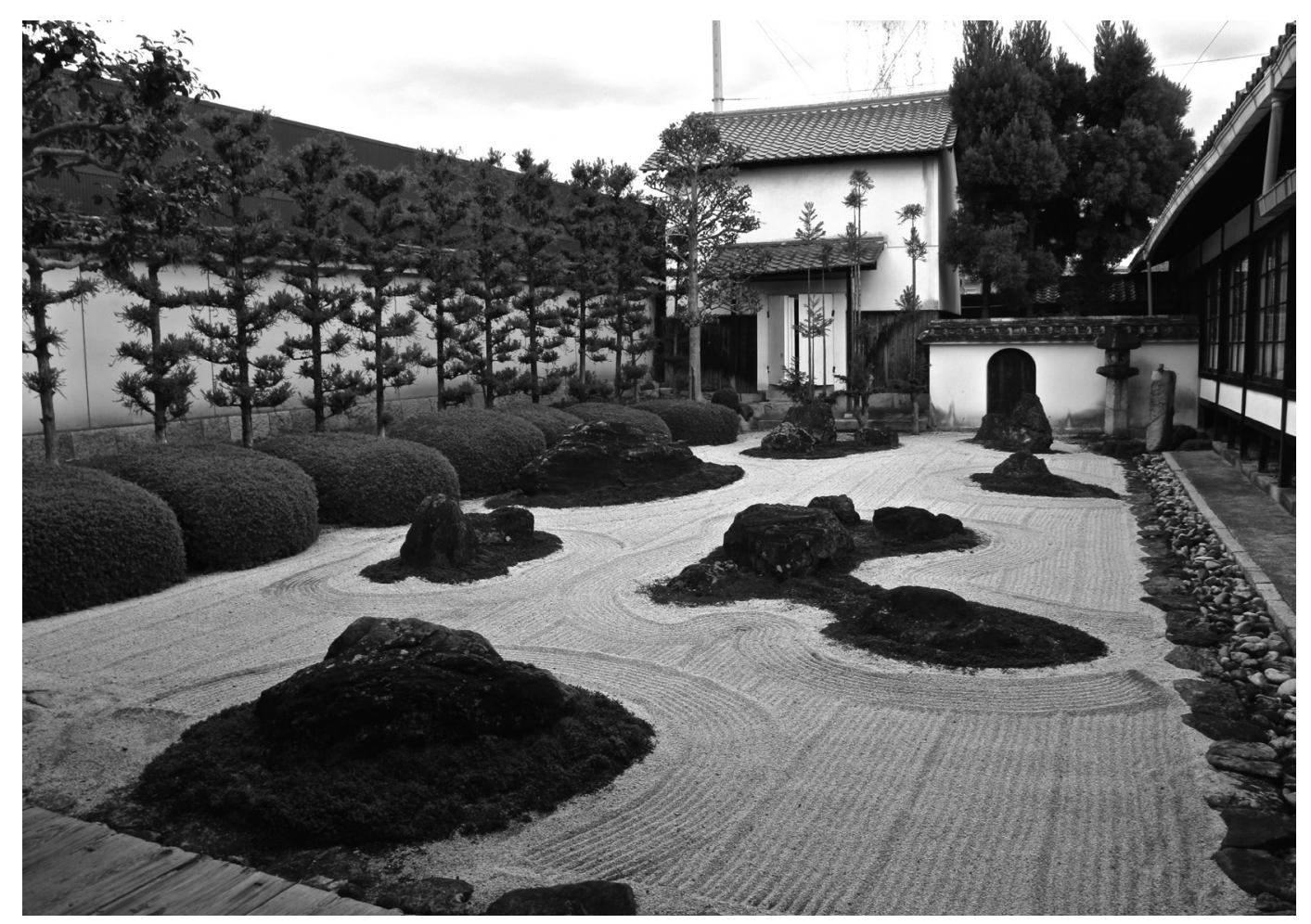

